\title{
Knockdown of cathepsin $L$ sensitizes ovarian cancer cells to chemotherapy
}

\author{
HONGMEI ZHANG ${ }^{1,2}$, LUOSHENG ZHANG ${ }^{2}$, LIXIA WEI $^{2}$, XINGWANG GAO ${ }^{2}$, \\ $\mathrm{LI} \mathrm{TANG}^{2}$, WEI GONG ${ }^{2}, \mathrm{NA} \mathrm{MIN}^{2}, \mathrm{LI} \mathrm{ZHANG}^{2}$ and YAWEI YUAN ${ }^{1}$

\begin{abstract}
${ }^{1}$ Department of Radiation Oncology, Nangfang Hospital, Southern Medical University, Guangzhou, Guangdong 510515;
\end{abstract} \\ ${ }^{2}$ Department of Oncology, The 458th Hospital of the People's Liberation Army, Guangzhou, Guangdong 510602, P.R. China
}

Received January 19, 2015; Accepted February 16, 2016

DOI: $10.3892 / 01.2016 .4494$

\begin{abstract}
Ovarian cancer is a leading gynecological malignancy associated with high mortality. The development of acquired drug resistance is the primary cause of chemotherapy failure in the treatment of ovarian cancer. To examine the mechanism underlying paclitaxel resistance in ovarian cancer and attempt to reverse it, the present study induced a TAX-resistant ovarian cancer cell line, SKOV3/TAX. Cathepsin L (CTSL) has been found to be overexpressed in ovarian cancer. The aim of the present study was to investigate the possible involvement of CTSL in the development of TAX resistance in ovarian cancer. CTSL expression was knocked down in SKOV3 ovarian cancer cells and their phenotypic changes were analyzed. The effects of silenced CTSL on the resistant cell line were investigated by proliferation and apoptosis analysis compared with control SKOV3 cells. CTSL was more highly expressed in SKOV3/TAX cells compared with SKOV3 cells. Paclitaxel treatment downregulated the expression of CTSL in SKOV-3 but not in the paclitaxel-resistant SKOV3/TAX cells. CTSL small hairpin RNA (shRNA) knockdown significantly potentiated apoptosis induced by paclitaxel compared with SKOV3/TAX cells transfected with control shRNA, suggesting that CTSL contributes to paclitaxel resistance in ovarian cancer cells and that CTSL silencing can enhance paclitaxel-mediated cell apoptosis. Thus, CTSL should be explored as a candidate of therapeutic target for modulating paclitaxel sensitivity in ovarian cancer.
\end{abstract}

\section{Introduction}

Ovarian cancer is the leading cause of gynecologic cancer-related mortality worldwide, as the majority of patients

Correspondence to: Professor Yawei Yuan, Department of Radiation Oncology, Nangfang Hospital, Southern Medical University, 1838 North Guangzhou Avenue, Guangzhou, Guangzhou, Guangdong 510515, P.R. China

E-mail: dryuanyawei@163.com

Key words: ovarian cancer, cathepsin L, paclitaxel, SKOV3/TAX cells, proliferation, apoptosis, chemoresistance present with advanced disease at diagnosis (1). The standard treatment for ovarian cancer is surgical cytoreduction and systemic chemotherapy, typically paclitaxel and platinum (2). Although improvement in median survival has been observed in recent decades, the majority of patients eventually succumb to recurrent, progressive disease due to resistance to chemotherapy (3). A combination of paclitaxel and carboplatin has widely been used as the first-line chemotherapy for patients with ovarian cancer. Paclitaxel acts specifically during the G2-M phase of the cell cycle by inducing abnormal spindles and disruption of microtubule dynamics, thereby blocking cell cycle progression. Despite its initial effectiveness as a cancer therapeutic agent, in the majority cases patients eventually become insensitive to paclitaxel-based chemotherapy and relapse (4). With the increasing emergence of paclitaxel resistance, the identification of suitable biomarkers for predicting chemosensitivity to paclitaxel may be key for improving the therapeutic outcome of patients with ovarian cancer.

Cathepsin L (CTSL), a lysosomal endopeptidase expressed in most eukaryotic cells, is a member of the papain-like family of cysteine proteinases (5). CTSL has a major role in antigen processing, tumor invasion and metastasis, bone resorption, and turnover of intracellular and secreted proteins involved in growth regulation (6). Increased CTSL levels have been identified in multiple tumor types and associated with short survival of several types of cancer. In addition to its well-established roles in development, growth and carcinogenesis, CTSL has been implicated in drug resistance (7-9). In a recent study, CTSL was overexpressed in ovarian cancer (10); however, no research regarding the association between CTSL with paclitaxel resistance in ovarian cancer has been performed thus far. In the present study, we hypothesized that high CTSL would be associated with intrinsic clinical drug resistance, manifesting as decreased time to disease progression/recurrence in patients with ovarian cancer.

\section{Materials and methods}

Cell lines. SKOV3 human ovarian adenocarcinoma and SKOV3/TAX paclitaxel-resistant human ovarian adenocarcinoma cells were purchased from the Cell Bank of the Chinese Academy of Sciences Institute (Shanghai, China). SKOV3 and SKOV3/TAX cells were cultured in RPMI-1640 
(Sigma-Aldrich, St. Louis, MO, USA) supplemented with $10 \%$ fetal bovine serum and $100 \mathrm{U} / \mathrm{ml}$ penicillin-streptomycin (Invitrogen; Thermo Fisher Scientific, Inc., Waltham, MA, USA). All cell lines were maintained at $37^{\circ} \mathrm{C}$ in humidified incubator with $5 \% \mathrm{CO}_{2}$. Cell culture medium was changed every 3-5 days depending on cell density. For routine passage, cells were split at a ratio of $1: 4$ when they reached $85-90 \%$ confluency.

Western blotting analysis. Cell samples were solubilized in sodium dodecyl sulfate (SDS) lysis buffer (Beijing Solarbio Science and Technology, Co., Ltd., Beijing, China) and the protein concentrations were detected using the BCA protein assay kit (Pierce Biotechnology, Inc., Rockford, IL, USA). Equal quantities of protein sample ( $30 \mathrm{mg} / \mathrm{lane})$ were separated by electrophoresis through $9.0 \%$ resolving SDS-polyacrylamide gel and then transferred to nitrocellulose membranes (Bio-Rad Laboratories, Inc., Hercules, CA, USA). The non-specific binding sites were blocked by immersing the membrane into $5 \%$ non-fat milk in Tris-buffered saline with $0.5 \%$ Tween-20 (TBS-T) solution (Beijing Solarbio Science and Technology, Co., Ltd.) for $1 \mathrm{~h}$, and then incubating the membrane with a primary mouse monoclonal anti-CTSL antibody (catalog no., sc-135859; dilution, 1:1,000; Santa Cruz Biotechnology, Inc., Dallas, TX, USA) for $2 \mathrm{~h}$ at room temperature (RT). After washing 3 times with TBS-T, the membranes were incubated with a HRP-conjugated secondary sheep anti-mouse IgG antibody (catalog no., NA9310-1ML; dilution, 1:1,000 in TBS-T; GE Healthcare Life Sciences, Chalfont, UK) for $1 \mathrm{~h}$ at RT. The membranes were washed and proteins were detected using an enhanced chemiluminescence system (GE Healthcare), according to the manufacturer's instructions. Mouse monoclonal anti-GAPDH antibody (catalog no., sc-365062; dilution, 1:5,000; Santa Cruz Biotechnology, Inc.) was used to confirm equal loading of lysates. ImageJ software (National Institutes of Health, Bethesda, MD, USA) was used to analyze the gray value.

Vector construction and transfection. A CTSL small hairpin RNA (shRNA) plasmid was purchased from Santa Cruz Biotechnology, Inc. (catalog no., sc-40685-SH; Dallas, TX, USA). Vector transfection was performed according to the manufacturer's protocol. SKOV3 and SKOV3/TAX cells were transfected with CTSL shRNA plasmid or empty vector (Control shRNA Plasmid-A; catalog no., sc-108060; Santa Cruz Biotechnology, Inc.) to knock down the expression of CTSL. Transfection was performed for $48 \mathrm{~h}$, according to the manufacturer's protocol.

3-(4,5-dimethylthiazol-2-yl)-2,5-diphenyltetrazolium bromide (MTT) reduction assay. Cells were seeded into 96-well plates at 2,000 cells/well. Each sample had four replicates. The cells were incubated with $0.2 \%$ MTT (Sigma-Aldrich) for $4 \mathrm{~h}$ at $37^{\circ} \mathrm{C}$. Subsequently, $100 \mathrm{ml}$ dimethylsulfoxide (Beijing Solarbio Science and Technology, Co., Ltd.) per well was added to the culture cells to dissolve the crystals, and cells were counted every day for 3 days by reading the absorbance at $490 \mathrm{~nm}$ (Synergy NEO; Bio-Tek Instruments, Inc., Winooski, VT, USA).

Annexin $V$ assay of chemoresistance. After $48 \mathrm{~h}$ of shRNA transfection, the cells were exposed to $100 \mathrm{~nm}$ paclitaxel
(6 mg/ml; Laboratório Químico Farmacêutico Bergamo Ltda., São Paulo, Brazil) for 24, 48 and 72 h. Then, cells were harvested, washed twice with ice-cold phosphate-buffered saline (Beijing Solarbio Science and Technology, Co., Ltd.) and suspended in Annexin V binding buffer (BD Biosciences, San Diego, CA, USA). The indicated amount of propidium iodide and Annexin V-fluorescein isothiocyanate (BD Biosciences) was added to the suspension and incubated for $20 \mathrm{~min}$ at RT in the dark. Subsequently, fluorescence was measured on a flow cytometer (BD FACSVerse 6 color; BD Biosciences).

Statistical analysis. Data analyses were performed using SPSS statistical software (version 15.0; SPSS, Inc., Chicago, IL, USA). Results are presented as the mean \pm standard deviation of three independent experiments. Comparisons of the percentage of viable cells and the number of apoptotic cells among groups were performed using the two-tailed Student's t-test. $\mathrm{P}<0.05$ was used to indicate a statistically significant difference.

\section{Results}

CTSL expression in SKOV3 and SKOV3/TAX cell lines. The results demonstrated that CTSL is highly expressed in SKOV3/TAX cells compared with SKOV3 cells (Fig. 1A). Considering this, SKOV3 and SKOV3/TAX cells were used to analyze the effect of paclitaxel treatment on the expression of CTSL. Cells were treated with paclitaxel (100 nM), and harvested at 24, 48 and $72 \mathrm{~h}$. Notably, immunoblotting demonstrated CTSL expression to be decreased at 48 and $72 \mathrm{~h}$ in SKOV-3 cells. However, CTSL expression remained relatively constant at high levels in SKOV3/TAX cells upon paclitaxel treatment (Fig. 1B). Thus, paclitaxel treatment downregulates the expression of CTSL in SKOV-3 cells but not in paclitaxel-resistant SKOV3/TAX cells, suggesting a role of CTSL in mediating paclitaxel resistance in ovarian cancer cells.

CTSL may affect the proliferation ability of ovarian cancer in vitro. To investigate the effect of CTSL on the proliferation ability of ovarian cancer cells, stable SKOV3 and SKOV3/TAX cell lines with downregulation of CTSL by shRNA were established (SKOV3-CTSL-shRNA and SKOV3/TAX-CTSL-shRNA, respectively). As indicated in Fig. 2A and B, the expression level of CTSL was markedly decreased in SKOV3-CTSL-shRNA and SKOV3/TAX-CTSL-shRNA cells compared with control cells (SKOV3-Con-shRNA and SKOV3/TAX-Con-shRNA, respectively). Next, the impact of CTSL silencing on cell proliferation was investigated. The results of the MTT assay demonstrated that knocking down CTSL in SKOV3 and SKOV3/TAX cells decreased cell proliferation (Fig. 3), suggesting that overexpression of CTSL may be involved in the development of ovarian cancer.

CTSL silencing promotes apoptosis induced by paclitaxel treatment in the resistant SKOV3/TAX cell line. To clarify the possible mechanisms involved in CTSL knockdown sensitizing cells to paclitaxel, an Annexin $\mathrm{V}$ apoptosis assay was performed. To this end, SKOV-3 and SKOV3/TAX cells were transiently transfected with control and CTSL shRNA, and 
A

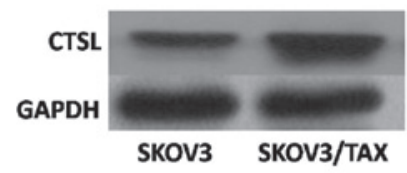

B $\operatorname{Tax}(100 \mathrm{nM})$

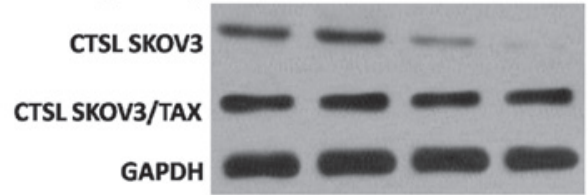

Figure 1. Western blots showing (A) expression of CTSL in SKOV3 and SKOV3/TAX cell lines; and (B) the effect of Tax treatment on the expression of CTSL in SKOV3 and SKOV3/TAX cell lines. CTSL, cathepsin L; Tax, Taxol (paclitaxel).

A
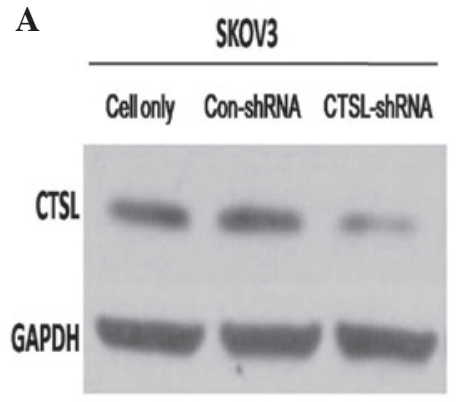

B
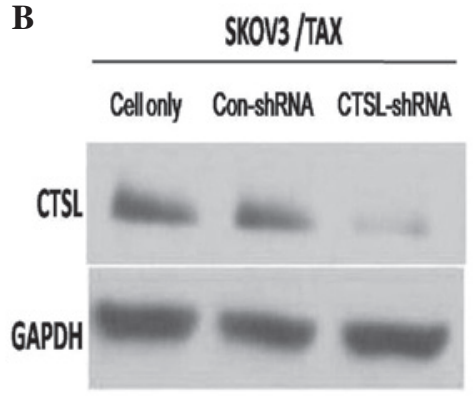

Figure 2. Western blots showing (A) significantly decreased expression levels of CTSL in SKOV3-CTSL-shRNA cells compared with Con cells (SKOV3-Con-shRNA); and (B) significantly decreased expression levels of CTSL in SKOV3/TAX-CTSL-shRNA cells compared with Con cells (SKOV3/TAX-Con-shRNA). Con, control; CTSL, cathespin L; shRNA, small hairpin RNA.

A

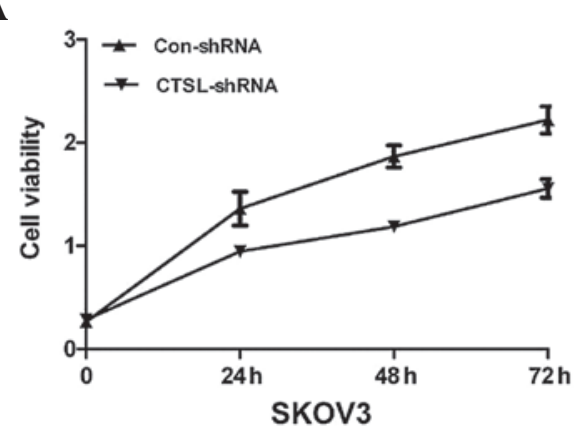

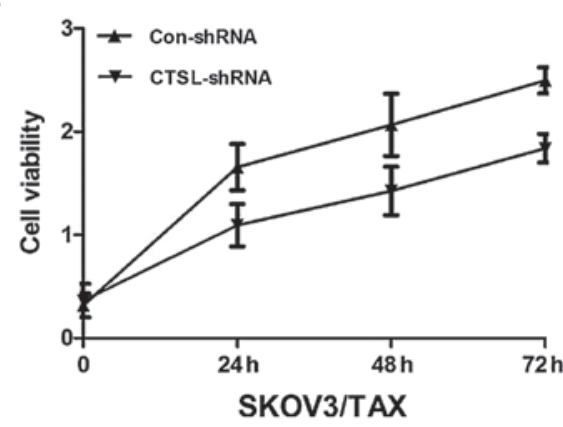

Figure 3. Knockdown of CTSL in (A) SKOV3 and (B) SKOV3/TAX cells causes decreased cell proliferation compared with Con cells. Cells were counted every day for 3 days by reading the absorbance at $490 \mathrm{~nm}$. Con, control; CTSL, cathespin L; shRNA, small hairpin RNA.
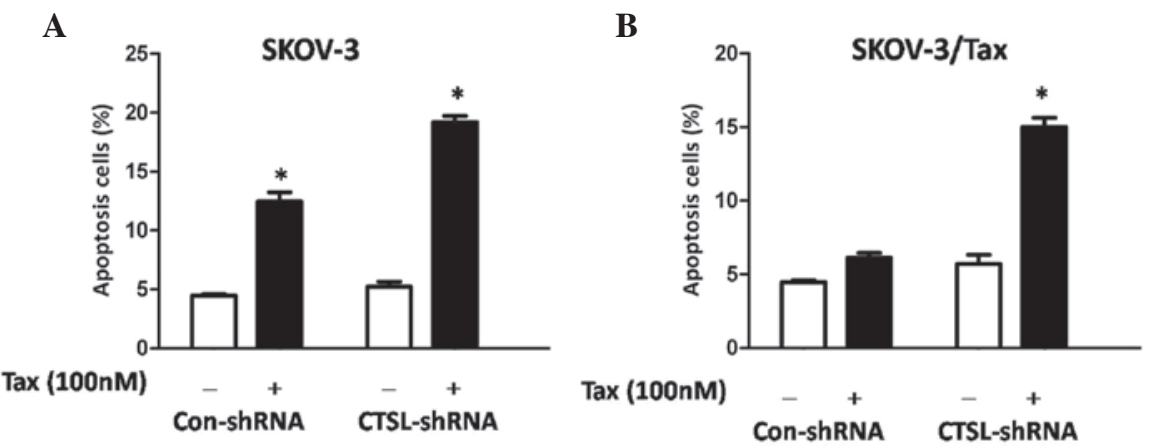

Figure 4. (A) Tax induced significantly potentiated apoptosis in SKOV-3 cells transfected with CTSL- or con-shRNA. "P $<0.05$ vs. no Tax treatment. (B) CTSL silencing significantly potentiated apoptosis induced by Tax in SKOV3/TAX cells compared with SKOV3/TAX cells transfected with Con-shRNA. ${ }^{*} \mathrm{P}<0.05$ vs. SKOV3/TAX-Con-shRNA cells treated with Tax. Tax, Taxol (paclitaxel); Con, control; shRNA, small hairpin RNA; CTSL, cathepsin L.

cultured in the presence or absence of paclitaxel $(100 \mathrm{nM})$ for $48 \mathrm{~h}$. The results revealed that paclitaxel induced significantly potentiated apoptosis in SKOV-3 cells transfected with CTSL or control shRNA, compared with equivalent untreated cells
( $\mathrm{P}=0.031$ and $\mathrm{P}=0.012$, respectively; Fig. 4A). Notably, it was observed that CTSL silencing significantly potentiated apoptosis induced by paclitaxel in SKOV3/TAX cells compared with SKOV3/TAX-Con-shRNA cells ( $\mathrm{P}=0.016$; Fig. 4B), suggesting 
that CTSL contributes to paclitaxel resistance in ovarian cancer cells and that CTSL silencing may enhance paclitaxel-mediated cell apoptosis. Depletion of CTSL in the SKOV-3 cells has no additive effect to paclitaxel treatment (Fig. 4A). This is likely due to the fact that paclitaxel functions through downregulating CTSL expression in SKOV-3 cells, as revealed by the western blot analysis.

\section{Discussion}

The majority of women with epithelial ovarian cancer present with advanced disease (stages III or IV) at the time of diagnosis. Current standard treatment of ovarian cancer, in both early and advanced stages, consists of complete cytoreductive surgery followed by chemotherapy, typically based on platinum and paclitaxel (11). However, the development of chemoresistance presents a major impediment for successful treatment. Of those patients with ovarian cancer, $>70 \%$ experience clinical remission after initial treatment, however, $60-75 \%$ of patients eventually relapse within 2 years of treatment (12). As paclitaxel is by far the most widely used microtubule-stabilizing agent in clinical treatment, the present study focused on for further characterizing paclitaxel-resistant cell lines.

The first observed function of CTSL in cancer progression was its ability to promote cancer metastasis (13). An early experimental study revealed that the metastatic capability of kidney and testicular tumor cells was correlated with CTSL activity (14). Furthermore, the finding that CTSL contributes to anti-apoptosis is also a well-accepted observation experimentally. Upon lysosomal membrane damage, CTSL is released into the cytosol where it cleaves BH3-interacting domain death agonist, disrupting the mitochondrial membrane and inducing apoptosis (15). CTSL is anti-apoptotic regardless of whether the induction of apoptosis triggers the intrinsic or extrinsic pathway, or even an indirect pathway via autophagy, upon treatment with arsenic trioxide (16). By contrast, other authors have highlighted the role of CTSL in conferring resistance towards chemotherapeutics and thus mediating an anti-apoptotic effect $(17,18)$. However, to date, little is known regarding the involvement of CTSL in drug-resistant ovarian carcinoma cells.

To determine an association between CTSL and chemoresistance, the present study employed a pair of established paclitaxel-sensitive and -resistant cell lines to analyze the effect of paclitaxel on CTSL. First, it was demonstrated that CTSL was more highly expressed in SKOV3/TAX cells compared with SKOV3 cells. Furthermore, CTSL expression decreased after 48 and $72 \mathrm{~h}$ of paclitaxel treatment in SKOV-3 cells, but remained relatively constant at high levels in SKOV3/TAX cells upon paclitaxel treatment, suggesting that the expression of CTSL mediated the resistance to paclitaxel in ovarian cancer cells. Thus, elevated levels of CTSL appear to correlate with lower drug susceptibility.

Previous studies have addressed the role of CTSL in conferring resistance towards chemotherapeutics and, thus, mediating an anti-apoptotic effect. In addition, CTSL silencing may inhibit the induction of the intrinsic apoptotic pathway in cancer cells via reduced CTSL nuclear activity, involving indirect p53 regulation of caspase 3/7 expression (19). CTSL was shown to be a critical molecule in paclitaxel resistance, however, the mechanism remained unclear. In the present study, SKOV-3 and SKOV3/TAX cells were transiently transfected with control and CTSL shRNA for $48 \mathrm{~h}$, and cultured in the presence or absence of paclitaxel $(100 \mathrm{nM})$ for $48 \mathrm{~h}$. The results showed that paclitaxel induced significantly potentiated apoptosis in SKOV-3 cells transfected with either CTSL or control shRNA. Notably, it was identified that CTSL silencing significantly potentiated apoptosis induced by paclitaxel in SKOV3/TAX cells with CTSL knockdown compared with SKOV3/TAX transfected with control shRNA, suggesting that CTSL silencing may enhance paclitaxel-mediated cell apoptosis.

Kenig et al partially revealed the mechanism of this apoptotic induction, demonstrating that CTSL inhibition lowers the apoptotic threshold of glioblastoma cells by upregulating p53 and caspase 3/7 expression (20).

The current findings demonstrate that CTSL knockdown can enhance the sensitivity of ovarian cancer cells to paclitaxel. Thus, CTSL should be explored as a candidate therapeutic target for modulating paclitaxel sensitivity in ovarian cancer.

\section{References}

1. Chen WQ, Zhang SW, Zou XN and Zhao P: Cancer incidence and mortality in China, 2006. Chin J Cancer Res 23: 3-9, 2011.

2. Vaughan S, Coward JI, Bast RC Jr, Berchuck A, Berek JS, Brenton JD, Coukos G, Crum CC, Drapkin R, Etemadmoghadam D, Friedlander M, et al: Rethinking ovarian cancer: Recommendations for improving outcomes. Nat Rev Cancer 11: 719-725, 2011.

3. Brouwer-Visser J, Lee J, McCullagh K, Cossio MJ, Wang Y and Huang GS: Insulin-like growth factor 2 silencing restores taxol sensitivity in drug resistant ovarian cancer. PLoS One 9: e100165, 2014.

4. Zhao F, Siu MK, Jiang L, Tam KF, Ngan HY, Le XF, Wong OG, Wong ES, Gomes AR, Bella L, et al: Overexpression of forkhead box protein M1 (FOXM1) in ovarian cancer correlates with poor patient survival andcontributes to paclitaxel resistance. PLoS One 9: e113478, 2014.

5. Goulet B, Baruch A, Moon NS, Poirier M, Sansregret LL, Erickson A, Bogyo M and Nepveu A: A cathepsin L isoform that is devoid of a signal peptide localizes to the nucleus in $\mathrm{S}$ phase and processes the CDP/Cux transcription factor. Mol Cell 14: 207-219, 2004.

6. Ruan J, Zheng H, Fu W, Zhao P, Su N and Luo R: Increased expression of cathepsin L: A novel independent prognostic marker of worse outcome in hepatocellular carcinoma patients. PLoS One 9: e112136, 2014.

7. Zajc I, Hreljac I and Lah T: Cathepsin L affects apoptosis of glioblastoma cells: A potential implication in the design of cancer therapeutics. Anticancer Res 26: 3357-3364, 2006.

8. Zheng X, Chu F, Chou PM, Gallati C, Dier U, Mirkin BL, Mousa SA and Rebbaa A: Cathepsin L inhibition suppresses drug resistance in vitro and in vivo: A putative mechanism. Am J Physiol Cell Physiol 296: C65-C74, 2009.

9. Primon M, Huszthy PC, Motaln H, Talasila KM, Torkar A, Bjerkvig R and Lah Turnšek T: Cathepsin L silencing enhances arsenic trioxide mediated in vitro cytotoxicity and apoptosis in glioblastomaU87MG spheroids. Exp Cell Res 319: 2637-2648, 2013.

10. Zhang L, Wei L, Shen G, He B, Gong W, Min N, Zhang L Duan Y, Xie J, Luo H and Gao X: Cathepsin L is involved in proliferation and invasion of ovarian cancer cells. Mol Med Rep 11: 468-474, 2015.

11. Jiang L, Siu MK, Wong OG, Tam KF, Lu X, Lam EW, Ngan HY, Le XF, Wong ES, Monteiro LJ, et al: iASPP and chemoresistance in ovarian cancers: Effects on paclitaxel-mediated mitotic catastrophe. Clin Cancer Res 17: 6924-6933, 2011.

12. Deraco M, Baratti D, Laterza B, Balestra MR, Mingrone E, Macrì A, Virzì S, Puccio F, Ravenda PS and Kusamura S: Advanced cytoreduction as surgical standard of care and hyperthermic intraperitoneal chemotherapy as promising treatment in epithelial ovarian cancer. Eur J SurgOncol 37: 4-9, 2011. 
13. Chauhan SS, Goldstein LJ and Gottesman MM: Expression of cathepsin L in human tumors. Cancer Res 51: 1478-1481, 1991.

14. Zhang W, Wang S, Wang Q, Yang Z, Pan Z and Li L: Overexpression of cysteine cathepsin $\mathrm{L}$ is a marker of invasion and metastasis in ovarian cancer. Oncol Rep 31: 1334-1342, 2014.

15. Stoka V1, Turk B, Schendel SL, Kim TH, Cirman T, Snipas SJ, Ellerby LM, Bredesen D, Freeze H, Abrahamson M, et al: Lysosomal protease pathways to apoptosis. Cleavage of bid, not pro-caspases, is the most likely route. J Biol Chem 276: 3149-3157, 2001.

16. Xu M, Yang L, Rong JG, Ni Y, Gu WW, Luo Y, Ishidoh K, Katunuma N, Li ZS and Zhang HL: Inhibition of cysteine cathepsin B and $\mathrm{L}$ activation in astrocytes contributes to neuroprotection against cerebral ischemia via blocking the tBid-mitochondrial apoptotic signaling pathway. Glia 62: 855-880, 2014

17. Wille A, Gerber A, Heimburg A, Reisenauer A, Peters C, Saftig P, Reinheckel T, Welte T and Bühling F: Cathepsin L is involved in cathepsin $\mathrm{D}$ processing and regulation of apoptosis in A549 human lung epithelial cells. Biol Chem 385: 665-670, 2004.
18. Walz M, Kellermann S, Bylaite M, Andrée B, Rüther U, Paus R, Kloepper JE, Reifenberger J and Ruzicka T: Expression of the human Cathepsin L inhibitor hurpin in mice: Skin alterations and increased carcinogenesis. Exp Dermatol 16: 715-723, 2007.

19. Levicar N, Dewey RA, Daley E, Bates TE, Davies D, Kos J, Pilkington GJ and Lah TT: Selective suppression of cathepsin L by antisense cDNA impairs human brain tumor cell invasion in vitro and promotes apoptosis. Cancer Gene Ther 10: 141-151, 2003.

20. Kenig S, Frangež R, Pucer A and Lah T: Inhibition of cathepsin L lowers the apoptotic threshold of glioblastoma cells by up-regulating p53 and transcription of caspases 3 and 7. Apoptosis 16: 671-682, 2011 